\title{
NONINVERTIBLE RETRACTS
}

\author{
JOE YANIK
}

\begin{abstract}
We demonstrate that if $P$ is a projective $R[X]$-module that is not stably extended from an $R$-module, then the symmetric algebra of $P$ over $R[X]$ is a retract of a polynomial ring over $R$, but is not an invertible $R$-algebra. Hence, there are noninvertible retracts over a quite general class of rings.
\end{abstract}

Introduction. Let $R$ be a commutative ring. There are two types of $R$-algebras that have at various times in the literature been defined to be projective: those which are a retract of a polynomial ring (which we will call, simply, retracts) and those which are a tensor factor of a polynomial ring (invertible). Some examples which are both retracts and invertible are polynomial rings and symmetric algebras of projective modules. For some time it seemed possible that they might be one and the same. In fact any invertible algebra is a retract. Recently, however, Greither [G] constructed examples of algebras that were retracts but not invertible. All of Greither's examples were algebras in one variable over a nonseminormal ring. In this article we show how to construct noninvertible retracts over any ring $R$ for which there is a projective $R\left[X_{1}, \ldots, X_{n}\right]$-module that is not stably extended from an $R$-module. Hence, using an example from [W] (it is actually an example due to Swan) we observe that there is even a UFD for which there is a noninvertible retract.

We do this by using Greither's technique of looking at the module of differentials. For a retract we note that the module of differentials is projective while the module of differentials of an invertible algebra is a projective module that is stably extended from an $R$-module. How far this goes towards characterizing these algebras is unclear at this time. (I.e. is there a noninvertible retract whose module of differentials is stably extended?)

Finally, we apply these techniques to some examples constructed in [Y] which prove to have the desired properties.

1. Preliminaries. All rings and algebras will be commutative and homomorphisms between $R$-algebras will be $R$-algebra homomorphisms.

Definitions. Let $R$ be a commutative ring and let $A$ and $B$ be $R$-algebras. A homomorphism $r: B \rightarrow A$ is an $R$-retraction of $B$ onto $A$ if there is a homomorphism $i: A \rightarrow B$ such that $r \circ i$ is the identity on $A$. Note that $i$ is necessarily one-to-one so that $B$ contains an isomorphic copy of $A$. In fact $B \cong A \oplus I$ where $I$ is the kernel of $r$. In this case $A$ is said to be an $R$-retract of $B$.

Received by the editors March 5, 1982 and, in revised form. April 27, 1982.

1980 Mathematics Subject Classification. Primary 13F20.

Key words and phrases. Retract, invertible algebra, symmetric algebra, module of differentials. 
If $A$ is an $R$-retract of some polynomial ring in finitely many variables over $R$ then we say that $A$ is an $R$-retract (or simply a retract when the ring is clear from the context).

$A$ is invertible if there is an $R$-algebra $B$ such that $A \otimes_{R} B \cong R\left[X_{1}, \ldots, X_{n}\right]$, a polynomial ring in finitely many variables.

$A$ is an augmented $R$-algebra if $R$ is an $R$-retract of $A$. In this case the kernel of the retraction mapping is called the augmentation ideal of $A$.

In [C] Connell observed that all invertible augmented $R$-algebras are retracts. We note here that all invertible algebras are augmented.

1.1. Lemma. Let $R$ be a commutative ring and $A$ an $R$-algebra.

(1) If $A$ is either invertible or a retract, then $A$ is an augmented $R$-algebra.

(2) If $A$ is invertible, then $A$ is a retract.

Proof. (1) Suppose $A \otimes_{R} B \cong R\left[X_{1}, \ldots, X_{n}\right]$. Then

$$
A \rightarrow A \otimes_{R} B \rightarrow R\left[X_{1}, \ldots, X_{n}\right] \rightarrow R
$$

provides the retraction, where $R\left[X_{1}, \ldots, X_{n}\right] \rightarrow R$ is the standard retraction sending the $X_{i}$ 's to 0 .

Similarly if $A$ is a retract of $R\left[X_{1}, \ldots, X_{n}\right]$ then $R\left[X_{1}, \ldots, X_{n}\right] \rightarrow R$ induces a retraction on $A$.

(2) If $A \otimes_{R} B \cong R\left[X_{1}, \ldots, X_{n}\right]$, then $A$ and $B$ are augmented (by (1)). If $B=R \oplus J$, then

$$
R\left[X_{1}, \ldots, X_{n}\right] \cong A \otimes_{R}(R \oplus J) \rightarrow A \otimes_{R} R \cong A
$$

is a retraction of $R\left[X_{1}, \ldots, X_{n}\right]$ onto $A$.

One can now ask the question: for what rings $R$ is it true that all retracts are invertible? (Or is it true for any rings at all?) Greither constructed some examples in which $R$ is nonseminormal. In the next section we show that, even when $R$ is factorial, there may be retracts that are not invertible.

2. The examples. Let $R$ be a commutative ring and $A$ an $R$-algebra. We begin with some observations about $\Omega_{A / R}$, the module of differentials of $A$ over $R$.

2.1. LeMma. If $A$ is an $R$-retract, then $\Omega_{A / R}$ is a projective $A$-module.

Proof. We may assume that, for some $n, A \subset R\left[X_{1}, \ldots, X_{n}\right]$ and there is a retraction mapping $r: R\left[X_{1}, \ldots, X_{n}\right] \rightarrow A$.

$A \rightarrow R\left[X_{1}, \ldots, X_{n}\right]$ induces an exact sequence $\left[\mathbf{M}\right.$, Theorem 57] with $R\left[X_{1}, \ldots, X_{n}\right]$ $=R[X]$ :

$$
\Omega_{A / R} \otimes_{A} R[X] \rightarrow \Omega_{R[X] / R} \rightarrow \Omega_{R[X] / A} \rightarrow 0 ;
$$

tensoring with $A$ we get

$$
\Omega_{A / R} \rightarrow \Omega_{R[X] / R} \otimes_{R[X]} A \rightarrow \Omega_{R[X] / A} \otimes_{R[X]} A \rightarrow 0 .
$$

But $r$ induces

$$
\Omega_{R[X] / R} \otimes_{R[X]} A \rightarrow \Omega_{A / R},
$$


which is easily seen to provide a splitting map for $(*)$. Hence,

$$
A^{n} \cong \Omega_{R[X] / R} \otimes_{R[X]} A \cong \Omega_{A / R} \oplus\left(\Omega_{R[X] / A} \otimes_{R[X]} A\right)
$$

and $\Omega_{A / R}$ is projective.

Definition. Let $A$ be an $R$-algebra, $M$ an $A$-module. Then $M$ is extended from an $R$-module if there is an $R$-module $M_{0}$ such that $M \cong M_{0} \otimes_{R} A$. (Note that if $A$ is an augmented $R$-algebra with augmentation ideal $I$, then $M_{0}$ is necessarily isomorphic to $M / I M$.)

$M$ is stably extended from an $R$-module if, for some $n, M \oplus A^{n}$ is extended from an $R$-module.

2.2. Lemma [G, 4.1]. If $A$ is an invertible $R$-algebra, then $\Omega_{A / R}$ is stably extended from a projective $R$-module.

(Greither actually did only the local case but the proof is essentially the same.)

Notation. Let $P$ be an $R$-module. Then we denote by $S_{R}(P)$ the symmetric algebra of $P$. If $P$ is projective, then $S_{R}(P)$ is invertible. In fact if $P \oplus Q \cong R^{n}$, then $S_{R}(P) \otimes_{R} S_{R}(Q) \cong R\left[X_{1}, \ldots, X_{n}\right]$. (See [Bo] or [E-H] for further discussion of symmetric algebras.)

The proof of the following lemma is routine and is omitted.

2.3. Lemma. Let $P$ be a projective $R$-module. $A=S_{R}(P)$. Then $\Omega_{A / R} \cong P \otimes_{R} A$.

2.4. THEOREM. Let $P$ be a projective $R\left[X_{1}, \ldots, X_{n}\right]$-module which is not stably extended from an $R$-module. Then $A=S_{R\left[X_{1} \ldots X_{n}\right]}(P)$ is an $R$-retract, but not $R$-invertible.

Proof. First note that by previous comments $A$ is $R\left[X_{1}, \ldots, X_{n}\right]$-invertible, hence a retract of some polynomial ring over $R\left[X_{1}, \ldots, X_{n}\right]$. Therefore it is an $R$-retract.

Let $R[X]=R\left[X_{1}, \ldots, X_{n}\right]$. Consider $R \rightarrow R[X] \rightarrow S_{R|X|}(P)=A$. This induces the exact sequence

$$
\Omega_{R[X] / R} \otimes_{R[X]} A \stackrel{v}{\rightarrow} \Omega_{A / R} \rightarrow \Omega_{A / R \mid X]} \rightarrow 0 .
$$

By [M. Theorem 57] $v$ has a left inverse if and only if any $R$-derivation of $R[X]$ into any $A$-module $T$ can be extended to an $R$-derivation from $A$ into $T$. Let $Q$ be such that $P \oplus Q \cong R[X]^{m}$. Then

$$
S_{R[X]}(P) \otimes_{R \mid X]} S_{R[X]}(Q) \cong R[X]\left[Y_{1}, \ldots, Y_{m}\right] .
$$

Since $A$ is a retraction of $R[X]\left[Y_{1}, \ldots, Y_{m}\right]$ any $A$-module is an $R[X]\left[Y_{1}, \ldots, Y_{m}\right]$ module. Given a derivation $D: R[X] \rightarrow T, D$ can easily be extended to $R[X]\left[Y_{1}, \ldots, Y_{m}\right]$ (simply define $D\left(Y_{i}\right)=0$ ). Restricting this extension to $A$ gives the desired result. Hence,

$$
\Omega_{A / R} \cong\left(\Omega_{R[X] / R} \otimes_{R[X]} A\right) \oplus \Omega_{A / R[X]} .
$$

But by Lemma $3, \Omega_{A / R[X]} \cong P \otimes_{R[X]} A$, so

$$
\Omega_{A / R} \cong A^{n} \oplus\left(P \otimes_{R[X]} A\right) .
$$


But this is not stably extended from $R$ since $P$ is not. In fact, if $A^{n} \oplus\left(P \otimes_{R[X]} A\right)$ $\oplus A^{l} \cong P_{0} \otimes_{R} A$, tensoring with $R[X]$ gives

$$
(R[X])^{n+l} \oplus P \cong P_{0} \otimes_{R} R[X] .
$$

2.5. Corollary. Let $R$ be a ring such that, for some $n$, the canonical injection $K_{0}(R) \rightarrow K_{0}\left(R\left[X_{1}, \ldots, X_{n}\right]\right)$ is not an isomorphism. Then there is an $R$-retract that is not invertible.

Proof. The hypothesis is equivalent to the existence of a projective $R\left[X_{1}, \ldots, X_{n}\right]$-module $P$ that is not stably extended from an $R$-module.

2.6. Corollary. Let $R=k[u, v, s, t] /\left(u^{3}-v^{2}-s t\right), k$ a field. Then $R$ is a UFD, but there is an $R$-retract that is not invertible.

Proof. By [W, Theorem 3.6], $R$ is a UFD such that there is a projective $R[X]$-module not stably extended from $R$.

\section{REFERENCES}

[B] H. Bass, Algebraic K-theory, Benjamin, New York, 1968.

[Bo] N. Bourbaki, Algebra. I, Hermann, Paris, 1970.

[C] E. H. Connell, A K-theory for the category of projective algebras, J. Pure Appl. Algebra 5 (1974), 281-292.

[E-H] P. Eakin and W. Heinzer, A cancellation problem for rings, Commutative Algebra, Lecture Notes in Math., Vol. 311, Springer-Verlag, Berlin and New York, 1973.

[G] C. Greither, Seminormality, projective algebras, and invertible algebras, J. Algebra 70 (1981), 316-337.

[M] H. Matsumura, Commutative algebra, 2nd ed., Benjamin \& Cumming, Reading, Mass., 1980.

[W] C. A. Weibel, K-theory and analytic isomorphism, Invent. Math. 61 (1980), 177-197.

[Y] J. Yanik, Projective algebras, J. Pure Appl. Algebra 21 (1981), 339-358.

Department of Mathematics, Louisiana State University, Baton Rouge, Louisiana 70803 